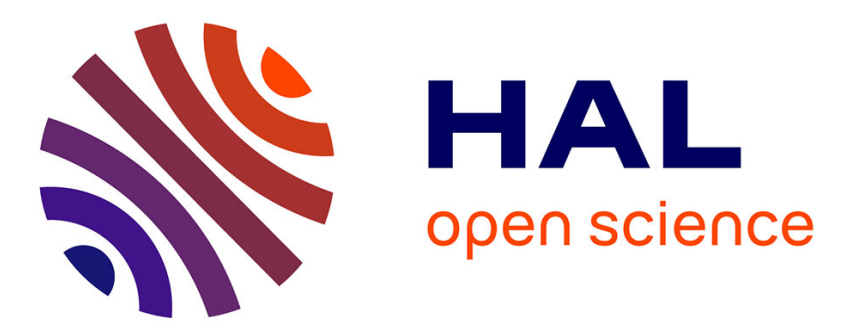

\title{
The lifetime of quasi-free exciton magnetic polaron in a quantum well with semimagnetic barriers
}

\author{
A. Kavokin
}

\section{To cite this version:}

A. Kavokin. The lifetime of quasi-free exciton magnetic polaron in a quantum well with semimagnetic barriers. Journal de Physique IV Proceedings, 1993, 03 (C5), pp.C5-79-C5-82. 10.1051/jp4:1993514 . jpa-00251600

\section{HAL Id: jpa-00251600 https://hal.science/jpa-00251600}

Submitted on 1 Jan 1993

HAL is a multi-disciplinary open access archive for the deposit and dissemination of scientific research documents, whether they are published or not. The documents may come from teaching and research institutions in France or abroad, or from public or private research centers.
L'archive ouverte pluridisciplinaire HAL, est destinée au dépôt et à la diffusion de documents scientifiques de niveau recherche, publiés ou non, émanant des établissements d'enseignement et de recherche français ou étrangers, des laboratoires publics ou privés. 


\title{
The lifetime of quasi-free exciton magnetic polaron in a quantum well with semimagnetic barriers
}

\author{
A.V. KAVOKIN
}

\author{
A.F. Ioffe Physico-Technical Institute, 194021 Politechnicheskaya 26, St. Petersburg, Russia
}

\begin{abstract}
The calculation of the radiative lifetime of a localized exciton confined in a quantum well is performed for CdTe/(Cd.Mn)Te structure where the quasi-free exciton magnetic polaron can be formed. There is shown that the polaron induced squeezing of the in-plane exciton localization radius leads to the increase of the lifetime. The lifetime dependence on the quantum well width is found to be altered by the polaronic effect in very thin CdTe/(Cd,Mn)Te quantum wells.
\end{abstract}

\section{Introduction}

The radiative lifetime of low-dimensional excitons has been a subject of theoretical and experimental studies during the recent decade(1-11). The cases of free two and one-dimensional excitons are substantially described theoretically( 1.2$)$ as well as the case of a bound zero-dimensional exciton $\left({ }^{3,4}\right)$.

The time-resolved photoluminescence spectroscopy being a direct way to measure the exciton radiative lifetime has been applied to study exciton states in various quantum wells $(7-11)$. The problem of adequate theoretical description of these experiments arises since there are not free but localized exciton states seen in the photoluminescence. The lifetime of the localized excitons is usually strongly different from that of free ones and depends, moreover, on the shape of the localization potential.

The subject of this paper is to study the localized exciton lifetime in semimagnetic heterostructures, where the exciton magnetic polaron (EMP) can be formed.The experimental studies of the dependence of $\tau$ on the quantum well (QW) width(7-11) reveal its strong deviation in different heterostructures. Following Ref. 7. it can be explained by the different values of the exciton Bohr radius in the studied materials. The simple calculation( $\left.{ }^{7}\right)$ shows that $\tau$ is proportional to the exciton area (squared exciton Bohr radius) divided by the area of the exciton inplane localization. The exciton in-plane Bohr radius, at, is a nonmonotonic function of the QW width, $L_{z}$. The corresponding variations of $\tau$ with $L z$ have been observed in GaAs/AlGaAs ( $\left.{ }^{7}\right)$ and InP/InGaAs $\left({ }^{8}, 9\right)$ QWs. The exciton Bohr radius appeared to be much less dependent on $L_{z}$ in wide gap II-VI heterosystems because of the higher electron and hole effective masses. This is a reason. probably, why very slight dependence of $\tau$ on $\mathrm{L}_{z}$ has been observed in CdTe/CdZnTe and CdTe/CdMnTe quantum wells $(10.11)$. To our knowledge, however, there are still no data on $\tau$ behaviour in ultrathin CdTe/CdMnTe QW5 where the exciton magnetic polaron (EMP) can be formed(12.13).

The purpose of this work is to study an effect of exchange induced in-plane squeezing of the exciton wave function on the radiative life time in $Q W 5$ with semimagnetic barriers, i.e. the EMP effect on $\tau$. In this sence we shell consider the 
polaron incluced changes of the exciton localization area with $L_{Z}$ variation - an effect which could be strons in semimagnetic sistems (12.13). We will show that the magnetic polaron effect can cause the dramatic increase of the exciton radiative life time in very thin CdTe/(Cd,Mn)Te Q ths.

\section{Theory}

Let us consider the exciton localized on an island of the QW width fluctuation in single QW with semimagnetic barriers like CdTe/(Cd.Mn)Te. Since the exciton is localized, we shell use the zeneral formulation for the lifetime, $x$ of zero-dimensional excitons obtained in Refs. 3-6. As follows from Refs. 5.6:

$$
\tau=3\left[\omega_{L T}\left(k a_{B}\right)^{3}\left(\int \phi\left(\vec{r}_{a}, \vec{r}_{h}\right) \delta\left(\vec{r}_{e}-\vec{r}_{h}\right) d \vec{r}_{c} d \vec{r}_{h}^{2}\right)\right]^{-1}
$$

where ${ }^{1} \mathrm{LT}$ and $\mathrm{a} \mathrm{B}$ are the sscillator strength and Bohr radius of an exciton in bulk well material: $k=\sqrt{\varepsilon_{B}} \frac{\omega}{C} \varepsilon_{B}$ is the dielertric constant supposed to be equal in well and barrier materials : to is the frequency of excitonic resonance : $\phi\left(r_{e}, r_{h}\right)$ is the exciton wave function, $r_{e}, r_{h}$ are the radius vectors of electron and hole.

Let us now discuss separately two limiting cases of the exciton in-plane localization: i) the case of independent electron and hole localization. $\left(R_{0}\left(a_{B}\right)\right.$ and ii) localization of an exciton as whole particle $\left.\left(R_{\sigma_{O}}\right\rangle_{a_{B}}\right)$, where $R_{0}$ is the typical size of the localization potential, $a_{B}$ is the exoiton Bohr radius.

In the former case. the exciton wave function can be represented as :

$$
\phi\left(\vec{r}_{e}, \vec{r}_{n}\right)=f_{e}\left(\rho_{e}\right) f_{h}\left(\rho_{h}\right) U_{e}\left(z_{e}\right) U_{h}\left(z_{h}\right)
$$

where $\left.f_{e i h}\right)\left(r_{e}(h)\right.$ ) and $T e(h)\left(z_{e}(h)\right.$ ) are the electron (hole envelope functions in and norral to the $Q W$ plare, respectively. Choosing $f_{e}(h)$ in exponential form, one can obtain from (1.):

$$
\tau=3\left[\omega_{L T}\left(k a_{b}\right)^{3} I_{c h}{ }^{2} \frac{16 \rho_{o a}^{2} \rho_{o h}{ }^{2}}{\left(\rho_{o c}+\rho_{o h}\right)^{4}}\right]^{-1},
$$

where

$$
I_{e h}=\int_{-\infty}^{\infty} U_{c}(z) U_{h}(z) d z
$$

Poe(h) is an electron (holel Bohr radius. In the case of exciton localization as a whole particle $\phi\left(r_{e}, r_{h}\right.$ ! can be written as:

$$
\phi=\mathrm{F}(\vec{R}) \mathrm{f}(\bar{\rho}) U_{\mathrm{e}}\left(\mathrm{z}_{\mathrm{e}}\right) \mathrm{U}_{\mathrm{h}}\left(\mathrm{z}_{\mathrm{h}}\right)
$$

where $F$ is the exciton in-plane envelope function, $f$ is a function of electron-hole relative motion. $R$ is the exciton centre of mass coordinate. $\rho=\rho_{e}-\rho_{h}$.

For exponential $F(R)$ and $f(\rho)$ one can obtain:

$$
\tau=3\left[\omega_{L \tau}\left(k a_{B}\right)^{3} I_{\text {ch }}^{2} \frac{16 L^{2}}{a_{\perp}^{2}}\right]^{-1} \text {. }
$$

The values of $\tau$ obtained in the approximation of exciton localized as whole particle are much smaller than that for the case of independently confined electron and hole becauze of the factor $\left(a_{\perp} / L\right)^{2}$. which is much smaller than unity in this case.It is seen now that in the case of exciton localization as a particle the dependece of $t$ on the QW width is given by factor $\frac{a_{\perp}^{2}}{I_{\sigma_{2} L^{2}}^{2}}$ in line with Ref. 7 . On the other hand. if electron 


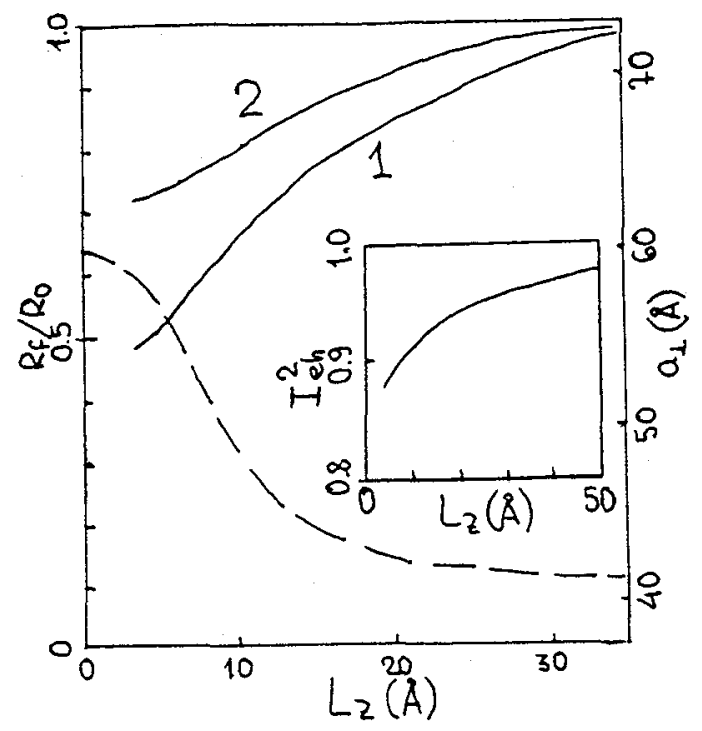

Fig. 1. - Exciton and hole inplane localization radius $R_{f}$ as function of the QW width (solid lines 1 and 2 , respectively). $R_{0}$ is the radius of an island of $Q W$ width monolayer fluctuation. Free exciton inplane Bohr radius, $a_{\perp}$, is shown by the dashed line. Inset shows the squared electron-hole overlap integrad. $\mathrm{I}_{\mathrm{eh}}{ }^{2}$ as function of QW width.

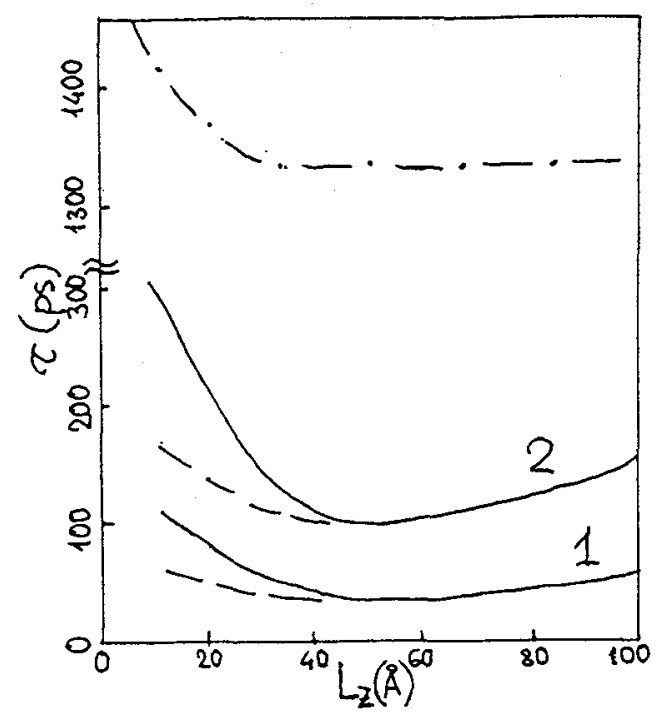

Fig. 2. - Exciton radiative lifetime, $\tau$ as function of QW width, $L_{z}$. Solid and dashed lines correspond to the case of exciton localization as a whole particle, dashed-dotted line - to the case of the independent electron and hole in-plane quantization. Curves 1 and 2 are calculated with exponential and Gaussian shape of the inplane exciton wave functions. The corresponding dashed lines show the calculation of $\tau$ which do not account the magnetic polaron effect.

and hole are quantized separately, one has the more complicated factor (see Eq. 3), which is, moreover, dependent on the choosen shape of electron and hole in plane wave functions.

To take into account the exchange induced exciton squeezing during the EMP formation in general we should find the dynamics of the radius of exciton (hole) localization $\mathrm{L}\left(\mathrm{P}_{\mathrm{Oh}}\right)$ in time. If the polaron formation time is larger or in order of the exciton life time one can expect the strongly nonexponential dependence of the exciton radiation intensity on time, so that $\tau$ as a parameter becomes hardly applicable to the system. That is why we shall limit ourself, for a while, by a consideration of the simplest case when the magnetic polaron formation time is much shorter than $\tau$. In this limit, to account the polaronic effect one just has to substitute $L\left(\rho_{\circ h}\right)$ in Eqs. $(5,3)$ by the corresponding radius of the magnetic polaron ground state.

The ground state of two-dimensional quasi-free EMP in a QW: with semimagnetic barriers has been calculated $(12,13)$ by the variational solving of the Schroedinger equation for in-plane exciton (or hole) motion.

The difference between the cases of exciton localization as a whole particle and separate confinement of electron and hole consists in the different values of in- 
plane mass and in-plane potential. The parameter Ro. which governs the potential should be taken different for an exciton and for a hole in order to obtain the magnetic polaron energy in agreement with the experimental data.

\section{Results and Discussion}

In this part the developed approach will be applied to the well-studied CdTe/CdMnTe system. Fig.1 shows the magnetic polaron induced decrease of the exciton localization radius in thin $\mathrm{CdTe} / \mathrm{Cd} 0.75^{\mathrm{Mn}} \mathrm{O} .25 \mathrm{Te}$ QWs calculated in the model of quasy-free EMP (12,13). We have used the parameters of the system from Refs.12.13 and the radius of initial exciton localization $R_{0}=85 \AA$. Solid curves $(1,2)$ represent the calculation with Gaussian and exponential trial functions, respectively. Dashed-curve shows the calculation in a model of independent electron and hole quantization with $R_{0}=35 \hat{A}$. One can see that in all the analysed cases the radius of exciton localization decreases substantially in the range of $Q W$ widths $L_{z}<30 \AA$, where the polaron can be formed. Dashed-dotted curve in Fig. 1 represents the exciton inplane Bohr radius behaviour with $\mathrm{L}_{\mathrm{Z}}$. Inset shows the very smooth dependence of the electron-hole overlap integral on $\mathrm{L}_{z}$.

The magnetic polaron induced decrease of the exciton localization area causes the sizeable enhancement of the exciton radiative life time, as Fig. 2 shows. The solid lines 1,2 represent the calculation of $\tau$ according to the Eq.5, i.e. for the cases of exciton localization as whole particle. The corresponding dashed lines show the calculated behaviour of $r$, if the polaron induced changes of the exciton localization area are neglected. The comparison of solid and dashed curves shows that the EMP formation causes 3-4 times stronger enhancement of $\tau$ in very thin $Q W_{5}$ than it is obtained from the variations of $a_{1}$. Dashed-dotted curve shows the exciton life time as function of $\mathrm{Lz}$-for the case of independent electron and hole in-plane quantizaion (Eq. 3).

The values of $\tau$ calculated in this model are of the same order as those calculated for bound zero-dimensional excitons (3.4). On the other hand, the experimentally measured exciton radiative life time in thick CdTe/CdMnTe QWs is about 150 ps (11), which clearly shows that the model of exciton in-plane localization as a particle much better corresponds to the experimentally studied samples.

\section{References}

1) Andreani L.C., Tassone F., Bassani F., Solid State Commun, 77, 641 (1991).

2) E.C. Ivchenko, Sov. Phys. Solid. State 33, 2388 (1991).

3) Al.L. Efros, Phys. Rev. B46, 7448 (1992).

4) J.M. Rorison, D.C. Herbert, Superlattices, \& Microstructures 1, 423 (1985).

5) E.L. Ivchenko, A.V. Kavokin, Sov. Phys. Solid. State, 34, 968 (1992).

6) E.L. Ivchenko, A.V. Kavokin, V.P. Kochereshko, P.S. Kop'ev, N.N. Ledentsov, Superlattices \& Microstructures, 12, 317 (1992).

7) J. Feldmann, G. Peter, E.O. Gobel, P. Dawson, K. Moore. C. Foxon and R.J. Elliott, Phys. Rev. Lett. 59, 2337 (1987).

8) U. Cebula, G. Bacher, G. Mayer, A. Forchel, W.T. Tsang, M. Razeghi, Superlattices \& Microstructures, 5, 227 (1989).

9) R. Sauer, S. Nilsson, P. Roentgen, W. Heuberger, V. Graf, A. Hangleiter, R. Spycher, Phys, Rev. B46, 9525 (1992).

10) R.P. Stanley, J. Hegarty, R. Fisher, J. Feldmann, E.O. Gobel, R.D. Feldman., R.F. Austin, Phys. Rev. Lett. 67. 128 (1991).

11) A. Pohlmann, R. Helmann, E.O. Gobel. D.R. Yakovlev, W. Ossau, A. Waag, R.N. Bichnell-Tassius, G. Landwehr, Appl. Phys. Lett., 61, 1929 (1992).

12) A.V. Kavokin. K.V. Kavokin. Semicond. Sci. \& Technology, 8, 191 (1993)

13) C. Benoit a la Guillaume. Phys. Stat. Sol. (b) 175, 369 (1993). 